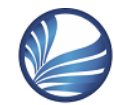

ELK

Asia Pacific Journals

www.elkjournals.com

\title{
HERD BEHAVIOUR AND AGGREGATE FLUCTUATIONS IN FINANCIAL MARKETS
}

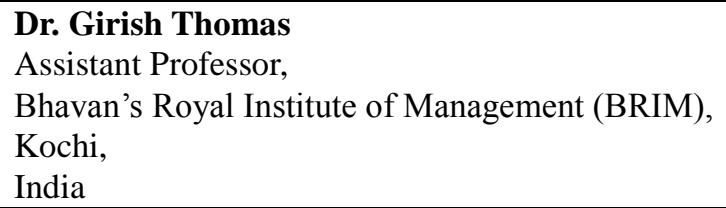

\begin{abstract}
We present a simple model of a stock market where a random communication structure between agents generically gives rise to a heavy tails in the distribution of stock price variations in the form of an exponentially truncated power-law, similar to distributions observed in recent empirical studies of high frequency market data. Our model provides a link between two well-known market phenomena: the heavy tails observed in the distribution of stock market returns on one hand and 'herding' behaviour in financial markets on the other hand. In particular, our study suggests a relation between the excess kurtosis observed in asset returns, the market order flow and the tendency of market participants to imitate each other.
\end{abstract}

Keywords: communication, market organization, random graphs.

\section{Introduction}

The studies based on empirical research related to the fluctuations in various financial assets' price derived that returns on stock distributions and changes in stock price comprise of fat tails that is liable to deviate from Gaussian distribution, especially in case of intraday time scales. Such fat tails, have been featured by the important excess kurtosis that remains persist after the mode of accounting in terms of heteroskedasticity among the derived data [9]. Observed heavy tails in such distributions remain in correspondence with larger counts of price fluctuations, "bursts" of volatility that is hard to illustrate in reference to variations noted within fundamental modes of economic variables.

The condition of significant fluctuations in terms of prices are in general not connected to data arrival or toward variations related to fundamental 
ELK

Asia Pacific Journals

www.elkjournals.com

economics. This can lead to the mode of thinking higher variability as in the returns of stock market that can stay in connection with collective phenomena like crowd effects or the instance of "herd" behaviour.

Though herding in case of financial markets has been noted to remain well documented on an empirical ground, some of the theoretical studies over the herding implications and imitation meant for statistical market properties get related to demand as well as price fluctuations. There are few questions, as how herding is liable to modify returns' distribution? What can be the significant implications related to herding for noting the connection among market variables, like order flow and the variability of price? These kinds of questions motivated the current study, undertaken by us.

This research aims to examine, the structure of a simple model, and the way through which the behaviour of the herd among market participants can get generically headed towards large fluctuations within aggregate excess of the demand, illustrated by heavy-tailed non-Gaussian distribution. Moreover, we analysed the empirically measurable quantities, like excess kurtosis meant for the returns as well as average flow of order that can stand in connection to each other in support of our model. The approach led by us, offers quantitative connection among two issues as stated above. These are heavy tails that has been observed in terms of stock market returns' distribution; and herd behaviour as observed within financial markets.

This article has five sections. These are 1) Section 1 is about reviewing the popular empirical facts related to heavytailed nature of stock returns' distribution and varied models as has been proposed to get in account of it.

2) Section 2 offer former empirical 
as well as theoretical work related to herding as well as imitation of financial markets that stands in connection with current study.

3) Section 3 leads the discussion about the statistical aspects related to the excessive demand caused by aggregation of huge count of random demands of the individuals in market.

4) Section 4 makes an illustration about our model and related analytical derivations.

5) Section 5 aims in interpreting economic results, compares the same in accordance to empirical data. This section also discusses possibility of extensions; illustrations about calculations (see Appendices).

1. The heavy-tailed nature of asset return distributions

It has been well realised that the distribution made over the returns of most of the financial assets, like, indexes,

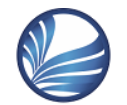

ELK

Asia Pacific Journals

$\underline{\text { www.elkjournals.com }}$ stocks and future investment exhibits slow asymptotic decay that is liable to deviate from normal distribution. This gets identified as a quantitatively reflected made in excess kurtosis that gets noted as:

$$
\kappa=\frac{\mu_{4}}{\sigma^{4}}-3
$$

Where $\mu 4$ represents the $4^{\text {th }}$ central moment and the symbol $\sigma$ represents the standard deviation of the returns. The representations of $\kappa$ is in terms of zero for gaining a normal distribution, yet ranges from 2 to 50 on daily returns and further remain higher in terms of intraday data. By means of an analytical research over the tails of distribution leads to an exponential decay in terms of most of the assets.

There are many statistical models that are considered in context of the heavy tails that are being observed under distribution of returns on asset. This can be exemplified by Mandelbrot's stable 
ELK

Asia Pacific Journals

www.elkjournals.com

paretian hypothesis which is an amalgamated form of distributions hypothesis, and respective models are in accordance to the conditional heteroskedasticity.

Due to the heteroskedasticity, there is this unconditional return's distribution that comprises heavy tails. Most of heteroskedasticity models are recognised as a return process that gets assumed for conditional Gaussian: that shocks "locally" noted Gaussian and nonGaussian feature related to the unconditional distribution as aggregation effect. It gets attained through superposing of huge count of local Gaussian shocks. In case of current illustration, sudden movements in terms of prices are interpreted in relation with high value marked for conditional variance.

It shows that though conditional heteroskedasticity is subject to lead to fat-tails under unconditional distributions, the relevant ARCH-type models are not liable to account over kurtosis of returns. However, on a theoretical ground, no priori reason can be marked in terms of postulating returns which are conditionally normal: though conditional normality attains convenient parameter estimation in reference to the resulting model, the distributions of nonnormal conditional aspect comprises of same qualitative features, in context of volatility clustering as accounting get better in the case of heavy tails. According to the report led by Gallant and Tauchen important evidence related to both conditional heteroskedasticity as well as conditional non-normality for daily NYSE value-weighted index are subject to illustrative analysis. In the same way, Engle and Gonzalez-Rivera declared that as $\operatorname{GARCH}(1,1)$ model gets implied for conditional variance in case of stock returns, conditional distribution attains enough kurtosis, 
ELK

Asia Pacific Journals

www.elkjournals.com

particularly in reference to small firm stocks. There are many scholars who proposed GARCH-type models along with non-normal conditional distributions.

Stable distributions is subject to give alternative towards heteroskedasticity in terms of generating respective fat tails, along with advantage of having natural interpretation related to aggregation over huge count of agent contributions to market fluctuations. This indeed, has got stable distribution that can be gained through the limitation of distributions of summations of independent or otherwise weakly dependent mode of random variables that is appropriately not liable to get shared through alternative models. However, unfortunately, properties of infinite variance related to these distributions never get observed by any empirical data. Sample variances can be noted that are not subject to increase indefinitely along with sample size, yet appears in terms of stabilizing determined value meant for enough data sets. We are hereby liable to make a discussion on distributions, illustratively in Section 3.

A very third approach that was advocated by Clark, refers to the model of stock returns through subordinated proceeding and remains typical to the subordinated state of Brownian motion. It is subject to stipulate by "stochastic time change" whereby one can transform complicated dynamics related to the price process in the form of Brownian motion or otherwise simple proceedings. In accordance to the selection of the subordinator, there is the room to gain wide-ranged distributions in terms of the increments that can comprise of heavy tails, which has positive excess kurtosis. It is important to note that stable distributions can be gained in terms of subordinated Brownian motion. Clark originally noted that the subordinator 


\section{2 \\ ELK \\ Asia Pacific Journals \\ www.elkjournals.com}

must have the trading volume. There are other choices that get proposed by various trades or otherwise through some local measures noted in the marketing activity. Still, none of these choices related to the subordinator is subject to lead towards normal mode of distribution in terms of increments related to the process of time-change, and further indicates huge count of fluctuations in terms of price that is not subject to complete the illustrated mode of large count of fluctuations in terms of trading trades.

In a nutshell, though heteroskedasticity as well as time deformation illustrate kurtosis related to asset returns, on a partial note; yet fail to explain the same quantitatively. This is noted even after the accounting gets marked for the related effects, whereby one remains at the left along with an important residual kurtosis in the transformed time series. Further, such approaches never rely over determined model in terms of market phenomenon that can generate data, which can be attempted to get illustrated. According to Bak, Paczuski and Shubik and Caldarelli, Marsili and Zhang, heavy tailed kind of return distributions marks as an emergent property in reference to market whereby the fundamentalist traders are subject to interact along with noise traders. As per the research led by Bak, Paczuski and Shubik, there are many trading rules as well as research works that consequently leads to statistical properties in case of time series meant for asset prices in every possible case. Computer simulations related to the model led by these scholars offered fattailed distributions in terms of asset returns that resembled qualitatively over empirical distributions in case of stock returns, leading to appearance meant for fat-tailed distributions. This can be considered as emergent property related to the large markets. Drawbacks noted 


\section{2 \\ ELK \\ Asia Pacific Journals \\ $\underline{\text { www.elkjournals.com }}$}

for this model are:

1) Firstly, it is a complicated model that has innumerable ingredients as well as parameters and remains hard to see the way each ingredient affects the obtained results. As a result the same diminishes explanatory power.

2) Secondly, complexity noted for this model never permits explicit calculations and relevantly prevent respective model as a means to compare with empirical values.

In the current research, we present an alternative proceeding that is noted through modelling communication framework among the market agents in the form of random graph, and radically proposes a simple mechanism that accounts for some non-trivial statistical features related to fluctuations of stock price. Though there are much rudimentary as well as comprising least ingredients rather than model as proposed by Bak, Paczuski and Shubik; yet the model considered by us allows analytic calculations and enables us to investigate and interpret the same under economic terms for the introduction of each of the parameters. We considered the basic intuition in a simple way. It is about the interaction made among the participants of the market by the imitation that can lead to large fluctuations under aggregate demand, offering heavy tails in case of returns' distribution.

2. Herd behaviour in financial markets Many recent research works aim to offer mimetic behaviour as the possible mode of illustrating excessive volatility in financial markets. By the application of herd behaviour in case of speculative markets, there is the derivation attained from few research works; as, by Scharfstein and Stein who evidenced herding in terms of behaviour related to fund managers. As per Grinblatt et al. herding in case of mutual fund's behavior 
and Trueman and Welch showed relevance of herding in terms of forecasts that has been made through financial analysts.

A noted theoretical side noted by various studies referred that, in case of market having noise traders, the instance of herd behavior never necessarily remain "irrational" in a way that he same can remain compatible added by the mode of optimizing behavior of respective agents. Some other motivations can get invoked in terms of illustrating markets' imitation, like "group pressure".

There are many models related to herd behavior that get noted in literature, and the very popular approach is by Banerjee and Bikhchan- dani et al. According to these models, people try to infer in terms of parameter that is noted from noisy observations, leading to decisions related to other agents, specifically managed by Bayesian approaches offering increase to "information cascades". A significant

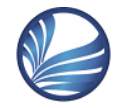

ELK

Asia Pacific Journals

www.elkjournals.com 
ELK

Asia Pacific Journals

www.elkjournals.com

comprises similar tendency towards the imitation of each other. In reference to aggregate variables, respective model refers to Gaussian distribution as the imitation gets weak, or otherwise towards bimodal distribution along with non-zero modes, that Orl'ean states in relation with collective marketing approach like, panics and crashes. In neither cases, there can be the attainment of heavy-tailed unimodal distribution at zero centre, as in noted stock returns.

This paper concentrates over the approach that stands different from the described approaches as noted above. The model selected by us is different from those in. There herding is not considered as sequential. Unrealistic nature can lead to results as noted in offering the result that all agents must get assumed in terms of imitating each other at the same degree.

We considered to avoid such issue by character of herd formation. random formation of groups related to agent, who are liable to imitate each other, yet the different agents' groups make independent decisions, that permits heterogeneous structure of the market. On a more specific note, our approach is managed about the interactions that has been noted among agents, with the result noted from a random communication framework and has been illustrated hereafter.

\section{Aggregation of random individual demands}

Considering stock market with $N$ agents, there is the labelling of integer as $1 \leq i \leq N$, whereby the trading remains as a single asset, that gets priced in the time $t$ and get denoted by $x(t)$. In every time period, a particular agent is offered with the scope to make a selection among either to buy stock, sell it or otherwise decide not to trade. Agent's stock demand is noted as $i$ that 
ELK

Asia Pacific Journals

www.elkjournals.com

gets represented through random variable

$\phi_{i}$, that can get valued as $0,-1$ or +1 :

whereby a positive value marked as $\phi_{i}$ is subject to represent "bull"- inclined to buy stock, and a negative value as "bear", eagerness to sell stock. It is here that $\phi_{i}$ $=0$ represents agent $i$ without any trade in the respective period. Random demands of the individual might remain either heterogeneous or under random resource of agents, or otherwise both. On an alternative manner, it can consequently lead to the application managed by agents related to simple rules of decision, whereby every group of agents follow some determined rules. Still, in terms of herding, we never gain explicit model for the process of decision that might lead towards individual demands as well as model leading to respective process of making decision under random variable $\phi_{i}$. As against this, there is scope to select from various binary models under the norms of microeconomics literature, we let an agent to remain inactive, means restricted from trading in respective time period $t$, illustrated below for derivations.

Let us consider simplification in respective time period, whereby an agent can either trade a asset unit or choose to remain inactive. Agent's demand $i$ represents $\phi_{i} \in\{-1,0,+1\}, \phi_{i}=-1$ for selling order. Aggregate demand of the excess for the noted asset at time $t$ thus, is

$$
D(t)=\sum_{i=1}^{N} \phi_{i}(t)
$$

where, algebraic nature is noted by $\phi_{i}$.Marginal distribution of the agent is noted from individual demand, assumed to remain symmetric as,

$$
P\left(\phi_{i}=+1\right)=P\left(\phi_{i}=-1\right)=a \quad P\left(\phi_{i}=0\right)=1-2 a
$$

in a way that average aggregate excess is liable to demand zero which notes fluctuation of market around equilibrium. Value of $a<1 / 2$ permits agents' finite 
ELK

Asia Pacific Journals

www.elkjournals.com

fraction for not trading in respective time.

We are trying to get a result that can be compared in terms of real data of market and short term excess towards the demand which cannot be observed easily. Moreover, various research works related to statistical features of financial series of time are made over returns, log returns or otherwise changes in pricing. Thus, we need to connect aggregate excess of the demand for a particular time in order to return or change price in respective period. Aggregate excess demand lays impact on stock price, and leads to increase in excess positive demand, in order to fall in case it remains negative. On a particular note, the compatibility noted in standard tatonnement ideas, gets assumed in terms of proportionality among the changed price (or return) and relevantly marked excess demand:

$$
\Delta x=x(t+1)-x(t)=\frac{1}{\lambda} \sum_{i=1}^{N} \phi_{i}(t)
$$

Where $\lambda$ is for assessing liquidity or market depth. It gets marked as the excess demand that must move price by one unit. The measurement of the price sensitivity towards fluctuations is noted in demand. Eq. (3), lays importance towards the impact of price of order flow against other causes related to fluctuations of price. Eq.(3) can be noted in absolute terms with $x(t)$ as price, or representing relative price variations; where $x(t)$ considers $\log$ of price and increment in terms of instantaneous return. Latter comprises of the advantage of offering guarantee towards price positivity, yet for short-run dynamics, where two specifications never differ substantially as two quantities comprises same empirical features. Same kinds of model for the impact of price in trades get noted by Hausman, Lo and MacKinlay. Though in case of long run economic concerns, against those with short term excess demand, there is the space to influence asset price evolution. 
ELK

Asia Pacific Journals

$\underline{\text { www.elkjournals.com }}$

This can lead to mean-reversion or otherwise complex behaviors, we lay emphasis over short-run price behaviors, as on intra-day time scales for stock markets. This is the reason that the counted approximation gets reasonable platform. Linear nature meant for this relation is subject to get interrogated. As a matter of fact, there are some empirical studies that shows that impact of price over trades may remain non-linear. At the onset, it is necessary to note that the selected literatures deal with impact of price over trades and not on flow of order (excess demand), that is comparatively harder for measurement. Consequences led by Farmer and co-workers as per the literature about the impact of price, blocks orders over different sizes that get sent towards market and relevantly indicate linear relationship in terms of smaller changes in price with increase in nonlinearity, as the blocks' size gets increased. Further, in case of return of one-period $\Delta x$ represents non-linear, yet smooth function $h(D)^{2}$ led by excess demand. This is followed by linearization of inverse demand function $h$ (first order Taylor expansion as $D$ ) noted in Eq.(3) that holds small fluctuations led by aggregate excess demand with $h^{\prime}(0)=1 / \lambda$.

For the evaluation of returns on stock distribution from Eq.(3), we must comprehend joint distribution led by individual demands $\left(\phi_{i}(t)\right)_{1 \leq i \leq N}$. Let's start by noting the simplest case whereby the individual demands $\phi_{i}$ related to different agents remain independent on an identical ground and distributed mode of random variables. We hereby refer to

\footnotetext{
${ }^{2}$ It is interesting to note that if $\Delta x=h(D)$, where $h$ is an increasing function of $D$ and if the individual demands $\left(\phi_{i}(t)\right)$ are sequences of independent random variables (a somewhat extreme assumption), then it is easy to show that the overall wealth of all traders increases on average with time.
} 
ELK

Asia Pacific Journals

www.elkjournals.com

the noted hypothesis as "independent agents" hypothesis. Here, joint distribution of individual demands are simple as the product of distributions and the variation of price $\Delta x$ summed up as $N$ iid with random variables having finite variance. As the terms noted in Eq.(3) remains large the central limit theorem gets implied to the summation as noted in Eq.(3). This tells us about the distribution of $\Delta x$, approximated by Gaussian distribution. As a matter of fact, the result holds as per demands of finite variance. This remains as a rationale meant for frequent application of normal distribution representing the model of distribution of returns on stock. In fact, if the variation of price of the market is noted as summation of large count of independent or otherwise weakly dependent mode of random effects, it gets plausible about the illustration of Gaussian description that must remain good.
Unfortunately, according to the empirical data distributions made over both the asset returns and changes in asset price are repeatedly noted for deviation in a significant way from Gaussian distribution, that exhibit fat tails as well as excess kurtosis.

However, independent agent model can generate aggregate distributions along with heavy tails. As a matter of fact, in case one relaxes about the assumption of individual demands as $\phi_{i}$, there is the finite variance as under the independent hypothesis (or weak dependence) related to individual demands, aggregate demand -and thus change in price in case Eq.(3) gets considered. There is also the room for stable (Pareto-Levy) distribution. The noted interpretation in context of stableParetian model was led by Mandelbrot [38] in context of heavy tails that get observed under increment distribution of diversified market prices. Infinite variance related to $\phi_{i}$ reflects market 


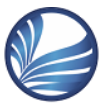

ELK

Asia Pacific Journals

$\underline{\text { www.elkjournals.com }}$

heterogeneity, as the broad wealth distribution of participants as marked by Levy \& Solomon.

Stable-Paretian hypothesis of Mandelbrot gets enough criticism, especially about the prediction of infinite variance related to stock returns implying practice related to sample variance, which is increases indefinitely along with sample size. This is a property that never gets observed under empirical data. On a precise note, analyses of the tails related to increment distribution meant for varied shows of financial assets with heavy tails, added by finite variance. Many distributions are subject to verify all such conditions; especially the example noted by various scholars, as an exponentially truncated stable distribution of the tails over density, followed by asymptotic form meant for exponentially truncated power law:

$$
p(\Delta x)_{|\Delta x| \rightarrow \infty}^{N} \frac{C}{|\Delta x|^{\mid+\mu}} \exp \left(-\frac{\Delta x}{\Delta x_{0}}\right)
$$

Exponent $\mu$ has been found to remain close to $1.5(\mu \simeq 1.4-1.6)$ in terms of a wide ranged stocks and indexes of the market. Such form of asymptotic aspect permits for heavy tails (or the excess kurtosis) without any kind of implementation over infinite variance.

However, central limit theorem holds in terms of some sequences of relevantly dependent variables: marked under mixing conditions. These are mathematical formulations related to the notion of being "weak" dependence, relatively aggregate mode of variables that gets distributed, in general. This is the reason that non- Gaussian and nonstable feature of empirical distributions, gain excess demand or returns on stock. It demonstrates failure of "independent agent", and shows an approach that is never close for being under good approximation. Dependence among 
ELK

Asia Pacific Journals

www.elkjournals.com

individual demands remains as an essential feature to the market structure and might not get left out within the aggregation procedure. This is not liable to get assumed to remain "weak" (in mixing condition) and d0 change the distribution leading to aggregate variable. In fact, assumption leads to the decisions of the agents that can be represented in terms of independent mode of random variables with highly unrealistic condition. This can be exemplified as an assumption that inclined to ignore necessary ingredient for the organized market, especially those related to interaction as well as communication between the agents.

In real markets, agents may form groups of various sizes, which then are liable to share data and act in accordance to relevant in coordination. For the financial market, traders are liable to align the trends of decisions and further act in terms of unison towards buying or selling; with a different interpretation related to the "group". This can be investment-oriented fund in correspondence to Firstly, in reference to a realistic structure of a market, there is the need to understand the complicated framework of clusters and further render the derived model in an analytical intractable manner. The derived consequence of the aggregate fluctuation pattern remains critical over specification of market structure.

Kirman suggested for an alternative way of gaining market communication framework related to itself in a stochastic manner. To generate random market framework, it is necessary to assume the participation in the market, especially in terms of meeting random trades caused as per the agent's wish to meet selling wishes. This approach of "random matching" by few scholars, gets considered in forming trading groups by Ioannides and for stock market model led 
ELK

Asia Pacific Journals

www.elkjournals.com

by Bak, Paczuski and Shubik. There is also the way of market participations forming groups or "clusters" by the modes of random process of matching, yet without trading noted within selected group. On the contrary, members of selected group follow common market strategy (as for instance, they decide in terms of buying or selling or no trade at all) and other groups can carry on with trade by centralized marketing process. For the financial market, the determined clusters can show that example of investors in mutual fund, something that is followed in this paper.

\section{Presentation of the model}

On a precise note, agents group in coalitions, group or clusters gain coordination from all the individual demands, in a way that every individual gains a cluster with similar belief related to the future movements of asset price. In the selected structure noted in preceding section, we noted all agents are part of offered cluster gain same demand $\phi_{i}$ in terms of stock. For the stock market, respective clusters is liable to remain in correspondence to mutual funds, for instance, whereby the managed portfolios by same fund manager or herding happening among security analysts. The RHS (right hand side) of equation (3) may thus, can be rewritten as the summation of clusters :

$$
\Delta x=\frac{1}{\lambda} \sum_{a=1}^{k} W_{\alpha} \phi_{\alpha}(t)=\frac{1}{\lambda} \sum_{a=1}^{n_{c}} X_{\alpha}
$$

where $W_{\alpha}$ represents the cluster size $\alpha, \phi_{\alpha}(t)$ the (common) individual demand of agents belonging to the cluster $\alpha, n c$ the number of clusters (coalitions) and $X_{\alpha}=\phi_{\alpha} W_{\alpha}$.

Coalitions can be considered by means of binary connection among agents, which can stand as a link among 
two determined agents, whereby they can undertake equivalent action over respective market, which refers to buying and selling of stock. For any agents with the pair of $i$ and $j$, let $p_{i j}$ remain as the probability, which comprises $i$ and $j$ linked together. Moreover, for the simplification, there can be the assumption of $p_{i j}=p$ as independent aspects for $i$ and $j$, whereby all the links remain equally probable. $(N-1) p$ referring to average count of agents, with a selected agent to remain connected to. As we inclined in researching $N \rightarrow \infty$ limit, $p$ must get selected as $(N-1) p$ being the finite limit. Natural selection is thus represented as $p_{i j}=c / N$, whereby any of the selection can get verified as per the condition mentioned above. It must remain asymptotically equivalent to the current one. Distribution made over the coalition sizes in market is completely specified within single parameter, $c$, and representing agents' willingness to align actions. This can be demonstrated as a coordination number that can assess clustering degree among agents.

Such framework gets termed as random graph in mathematical declarations: whereby the theory of random graph gets considered in context of the agents being with vertices of random graph related to the size $N$, and coalitions being connected graphical components. This kind of communication approach in terms of markets gets noted by the application of random graphs that has been initially suggested in literatures of economics, as by Kirman for understanding the core features of the large economy. Random graphs get used in multilateral matching in search for problems led by Ioannides. As per a good review over the applications related to the theory of random graph in terms of economic modelling see.

Features of large random graphs as in $N \rightarrow \infty$ gets limited and initially was 
ELK

Asia Pacific Journals

www.elkjournals.com

studied by Erd"os and Renyi. There is an extensive review led by mathematical results over random graphs. Core results related to combinatorial proceeding are noted in Appendix 1. It is by that $c=1$, where the density of the probability for distribution of the cluster size decreases asymptotically as per power law:

$$
P(W) \underset{W \rightarrow \infty}{\sim} \frac{A}{W^{5 / 2}}
$$

while values related to $c$ close remains < $1(0<1-c<<1)$, where size of cluster distribution gets cut off through exponential tail:

$$
P(W) \underset{W \rightarrow \infty}{\sim} \frac{A}{W^{5 / 2} / 2} \exp \left(-\frac{(c-1) W}{W_{0}}\right)
$$

For $\mathrm{c}=1$, respective distribution gains infinite variance, as $c<1$ and the variance turns finite, due to exponential tail. For such an instance, average coalition size for order $1 /(1-c)$ as well as average count of clusters remains as order $N(1-c / 2)$.

Coordination parameter represented by $c$ close to 1 represents every agent who tend for the establishment of a link along with other agent, and is noted as reasonable assumption.

This never rule out the creation of large coalitions by successive mode of binary connection among agents, yet prevents single agent from creating multiple links, as in case of centralized communication framework, where a single agent (the "auctioneer") gets linked to all other agents. Kirman stated that presence of a Walrasian auctioneer corresponds is related to such 'star-like', centralized, kind of communication framework. Thus, we exclude situation noted by construction: whereby we remain interested in market of distributed data and not about the centralized feature. This keeps in correspondence with situations noted in real markets. On a precise note, local market structure can get featured by following consequences [8]: wherein the limit $N \rightarrow \infty$, number $v_{i}$ 


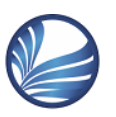

ELK

Asia Pacific Journals

www.elkjournals.com

of the neighbors of the selected agent $i$ remains as a Poisson random variable along with parameter $c$ :

$$
P\left(v_{i}=v\right)=e^{-c} \frac{c^{v}}{v !}
$$

Walrasian auctioneer $w$ can get linked to every agent: $v_{w}=N-1$. Probability for Walrasian auctioneer is noted as $N P\left(\nu_{w}=N-1\right) \quad$ leading to zero, as $N \rightarrow \infty$.

In context of a given cluster of market, there is the feature of size $W_{\alpha}$ as well as its'nature' of if the members are noted as buyers or sellers. This is specifically noted as variable $\phi_{\alpha} \in\{-1,0,1\}$. It remains reasonable in order to assume $W_{\alpha}$ and $\phi_{\alpha}$ being independent random variables. The group sizes never influence respective decision of selling or buying. The variable $X_{\alpha}=\phi_{\alpha} W_{\alpha}$ remains symmetrically distributed along with mass of $1-2 a$ at origin. Let

$$
F(x)=P\left(X_{a} \leq x \mid X_{a} \neq 0\right)
$$

Then distribution of $X_{\alpha}$ gets noted as -

$$
G(x)=P\left(X_{\alpha} \leq x\right)=(1-2 a) H(x)+2 a F(x)
$$

where $H$ represents unit step function at 0 (that is Heaviside function). We thus assume $F$ with continuous density, $f$. $f$ followed by decays asymptotically as noted in (6):

$$
f(x) \underset{|x| \rightarrow \infty}{\sim} \frac{A}{|x| 5 \mid / 2} e^{\frac{-(c-1) \mid x}{W_{0}}}
$$

Expression for variation of price $\Delta x$ thus reduces to the summation of $n_{r}$ iid as random variables $X_{\alpha}, \alpha=1 . . n_{c}$ along with heavy-tailed distributions (6):

$$
\Delta x=\frac{1}{\lambda} \sum_{\alpha=1}^{n_{\varepsilon}} X_{\alpha}
$$

Since the probability density of $X_{\alpha}$ attains the finite mass of $1-2 a$ at zero, it is only fraction $2 a$ of (5) that are represented by non-zero; count of non-zero terms is noted in respective order 
ELK

Asia Pacific Journals

www.elkjournals.com

$2 a \overline{n_{c}} \sim 2 a N(1-c / 2)=N_{\text {order }}(1-c / 2), \quad$ where

$N_{\text {order }}=2 a N$ represents average count of market participants that remains active in terms of trading in market in the determined time span. As for instance, $N_{\text {order }}$ is the count orders that gets attained in the noted span of time $[t, t+$ 1], whereby we assume various orders in correspondence to the determined net demands, that has been defined already about various agents' clusters. For determined span of time, may be for 15 minutes over liquid market, like NYSE, $N_{\text {order }}=100-1000$, there is the specific magnitude.

Distribution of price variation $\delta x$ gets noted as in -

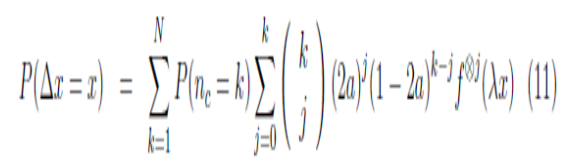

where $\otimes$ represent convolution product, whereby $n_{c}$ is the count of clusters. Aforementioned equation is liable to calculate generating functions $F$ related to aggregate excess noted over demand $D$

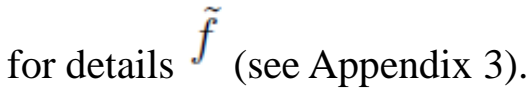

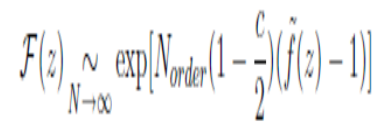

The aspects related to $D$ (and to $\Delta x$ ) can be gained by Taylor expansion over Eq.(12) (see Appendix 4). Calculation related to variance as well as fourth moment offers:
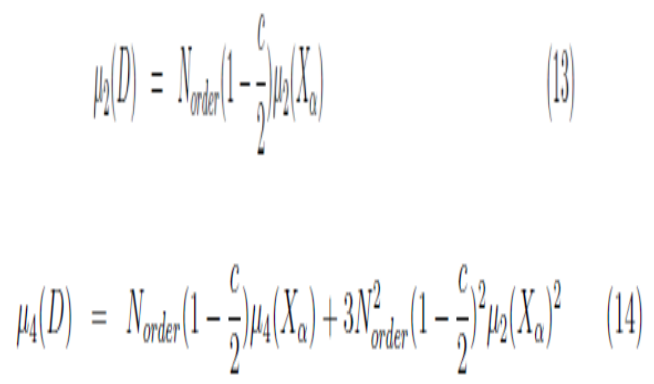

Interestingly, kurtosis' quantity of asset returns as in our model, must remain equal to kurtosis of excess demand $\kappa(D)$ :

$$
\kappa(D)=\frac{\mu_{4}\left(X_{\alpha}\right)}{N_{\text {order }}\left(1-\frac{c}{2}\right) \mu_{2}\left(X_{\alpha}\right)}
$$

Moments of $\mu j\left(X_{\alpha}\right)$ can be gained through expansion in $1 / \mathrm{N}$ whereby $\mathrm{N}$ 
represents count of agents in respective market (see Appendix 2). This is liable to substitute expression over the aforementioned formula offering kurtosis $\kappa(D)$ being a function to $c$ and relevant order flow:

$$
k(D)=\frac{2 c+1}{N_{\text {order }}\left(1-\frac{c}{2}\right) A(c)(1-c)^{3}}
$$

where $A(c)$ gets noted as normalization constant considering value that is close to 1 as in Appendix 2, trying to get finite limit as $c \rightarrow 1$. This is a relation that can be interpreted as: reduction volume of order flow leading to larger fluctuations in price, featured by larger excess of kurtosis. As a result, the correspondence the large fluctuations of price being inclined to occur within less active in the markets, featured smaller flow of order. It remains consistent along with results attained from different market microstructure models that have larger flow of order and enables easier mode of demand and supply regulation by market maker. Interestingly, we derived same qualitative feature in this approach and though we have not integrated explicitly the market maker in this model, the result gets compared to observation. The derived accounting is noted for heteroskedasticity, with conditional distribution made over stock returns meant for smaller firms being higher than large firms are. Stocks of small firm get featured by smaller flow of order $N_{\text {order }}=2 a N$, and the same gets compatible along our results.

On a significant note, Eq. (15) refers to kurtosis to be very large, even though number of orders is itself large, only if $c$ is close to 1 . As $A(1)$ is found to remain close to $1 / 2$, one can derive same for $c=$ 0.9 and $N_{\text {order }}=2 a N=1000$, where kurtosis $\kappa$ still remains as an order 10 , and has been noted as very active market over determined span of time intervals of 10 minutes. In fact, irrespective of $2 a N$ being not too large, asymptotic behaviour 
ELK

Asia Pacific Journals

www.elkjournals.com

related to $P(\Delta x)$ remains as a form noted in Eq. (6). The current model leads to determined value in a natural way of $\mu=$ $3 / 2$, that remains close to observed value of the real markets. Here, the value noted for $c$ can remain time dependent. As for instance, herding tendency inclines to remain stronger in an uncertain duration that leads to increased state of kurtosis. As $c$ reaches 1 , finite fraction among the market shares increases simultaneously with equivalent opinion and thus a crash. Extension of determined model can be the one that is related to market structure in respect to time evolution and remains explicitly modelled, and there can be possible feedback about the movement of the price moves over behavior of determined participants of the market.

\section{Discussion}

We tried to exhibit a stock market model that remains albeit its simplicity, and increase towards non-trivial probability mode of distribution related to aggregate excess of demand as well as variation in the price of the stock. This remains same as empirical distributions made over asset returns. The model considered by us demonstrates that as the naive model of the market refers to agents for not interacting, there is the tendency to rise distributed aggregate fluctuations normally. For this state of interaction among the market participants by rudimentary 'herding' mechanism offers quantitatively compared empirical derivations over stock distribution of market returns.

Our model derived the prediction about the fatness of asset returns' tails as measured through excess count of kurtosis as well as herding degree among participants of market assessed by parameter $c$, as in Eq.(15) .

Though we have assumed implicitly about $t$ for chronological time, the model can note $t$ as "market time", followed by 


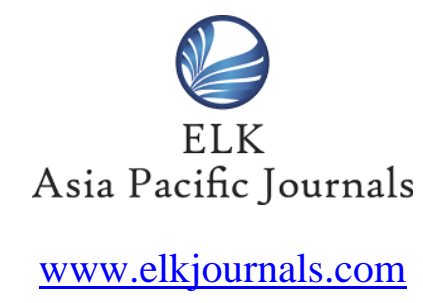

subordinated real time. Here the

economic system with self-organized difference is the underlying approach criticality.

without keeping Gaussian random walk.

Our model came up with questions, like value $c$ being specified as less than, and relevantly close to 1 . "Fine-tuning" is for some value that can be arbitrary until the same gets justified as assumption. Extension of our model can be related to time evolution for market framework that can be explicitly modelled in a way that the parameter $c$ can get critical region (close to 1).

An issue can be through "self-organized criticality" by Bak et al, whereby some dynamical systems can be generically evolved to determined state whereby parameters converge critical values towards scaling laws as well as heavytailed distributions meant for quantities as modelled. This can be met asymptotically and for an attractor, system dynamics noted by Bak, Chen, Scheinkman and Woodford refer simple
For the aforementioned result, there is no need to hold or adjust $c$ towards critical value, and the same is sufficient for $\mathrm{c}$ within determined value range. As $c$ approaches 1, Respective clusters turn larger and leads to giant coalition, as $c \geq$ 1. Activation of our model for such cluster can cause market crash. Realistically, dynamics of $c$ must be related to crash, yet not stable and giant cluster disaggregates get noted shortly after the same gets formed, preceded by short panic, the respective market resumes its normal activity. Mathematically, dynamics of $c(t)$ must get specified in context of value $c=1$ being 'repulsive'. This must be met by introducing effective feedback over prices on behaviour of participants of market. Here, nonlinear coupling can be noted among the trend of leading control mechanism $c$ within critical region. 
Another dynamical specification that remains compatible with our model is from agents having "threshold response" that was noted initially for collective phenomena in terms of economic systems. Heterogeneity gets allowed through individual threshold $\theta i$ noted by random variables. As for instance, there can be the assumption of $\theta i$ s to be iid by standard deviation $\sigma(\theta)$. To introduce interactions between agents by aggregate variable, every agent can observe aggregate excess demand $D(t)$ as in Eq.(2) or $D(t)+E(t)$, with $E$ as an exogeneous variable. Evolvement of agents thus follows agents that changes market position $\varphi(t)$ ("flips" from the long term to short term and vice versa) in case observed signal $D(t)$ crosses respective threshold $\theta i$. Noted aggregate fluctuations can happen by cascades or "avalanches" related to market flipping as per market positions related to agents' groups. It gets researched as per physical systems by Sethna et al. These scholars showed wide range of values related to $\sigma(\theta)$ with aggregate fluctuations, whereby distribution is made over power-law behavior along with exponential tails as per Eq.(6).

All these issues are illustrated hereafter in this work.

\section{References}

[1] Bak P., Tang C. \& Wiesenfeld, K. (1987)"Self-organized criticality" Physical Review Letters, 59, 381.

[2] Bannerjee, A. (1992)"A simple model of herd behavior" Quarterly Jour- nal of Economics, 107, 797-818.

[3] Bannerjee, A. (1993)"The economics of rumours" Review of Economic Studies, 60, 309-327.

[4] Billingsley, P. (1975) Convergence of probability measures, New York: Wiley.

[5] Bollerslev T., Chou R.C. \& Kroner K. (1992)"ARCH modeling in finance" Journal of Econometrics, 52, 5-59. 


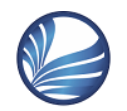

ELK

Asia Pacific Journals

www.elkjournals.com

[6] Bouchaud J.P. \& Potters M. (1997) Th'eorie des risques financiers, Paris: Ala Saclay.

[7] Caldarelli G., Marsili M. \& Zhang Y.C. (1997)"A prototype model of stock exchange" Universit'e de Fribourg preprint.

[8] Clark, P.K. (1973)"A subordinated stochastic process model with finite variance for speculative prices" Econometrica, $41,135-155$.

[9] Cont R., Potters M. \& Bouchaud J.P. (1997)'Scaling in stock market data: stable laws and beyond" to appear in Scale invariance and beyond, Proceedings of the CNRS Workshop on Scale Invariance (Les Houches, France), March 1997.

[10] Cutler D.M., Poterba J.M., \& summers, L. (1989)"What moves stock prices?" Journal of Portfolio Management, spring, 412.

[11] Engle, R. \& Gonzalez-Rivera (1991) "Semiparametric ARCH models" Journal of Business and Statistics, 9, 345-360
[12] Engle, R. (1995) ARCH: selected readings, Oxford: Oxford University Press.

[13] Erd"os, P. \& Renyi, A. (1960)"On the evolution of random graphs" Publications of the Mathematical Institute of the Hungarian Academy of Sciences, 5, 17-61.

[14] Fama, E.F. (1965)'"The Behavior of Stock market Prices" Journal of Business, 38, 34-105.

[15] Farmer, D. (1997), Seminar presented at Universit'e de Paris VI.

[16] Feller, W. (1950) Introduction to Probability theory and its applications, II, 3rd ed., New York: John Wiley \& Sons.

[17] Gallant, A.R. \& Tauchen, G. (1989)"Semi non-parametric estimation of conditional constrained heterogeneous processes", Econometrica, 10911120.

[18] Geman, H. \& An'e, T. (1996) "Stochastic subordination" RISK, Septem- ber.

[19] Granovetter, M. \& Soong, R. (1983) " Threshold models of 


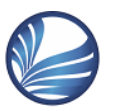

ELK

Asia Pacific Journals

www.elkjournals.com

diffusion and collective behavior"

Journal of Mathematical

Sociology, 9, 165-179.

[20] Grinblatt M., Titman S. \& Wermers R. (1995)"Momentum investment strategies, portfolio performance and herding: a study of mutual fund behavior" American Economic Review, 85 (5), 1088-1104.

[21] Guillaume D.M., Dacorogna M.M., Dav'e R.R., Mu“ller U.A., Olsen R.B., Pictet O.V. (1997) "From the birds eye to the microscope: a survey of new stylized facts of the intra-day foreign exchange markets" Finance and Stochastics, 1, 95130.

[22] Hausman J., Lo A.W. \& MacKinlay C. (1992)"An ordered probit anal- ysis of transaction stock prices" Journal of financial economics,31, 319- 379.

[23] Hsieh, D.A. (1991) "Chaos and non-linear dynamics: application to fi- nancial markets" , Journal of Finance, 46, 1839-77.

[24] Ioannides, Y.M. (1990) "Trading uncertainty and market form" Inter- national Economic review, 31, 3, 619-638.

[25] Ioannides, Y.M.

(1996) "Evolution of trading structures" in Arthur,B.W., Lane,D. \& Durlauf, S.N. (eds.) The Economy as an evolv-ing complex system (Santa Fe Institute), Addison Wesley.

[26] Kempf, A. \& Korn, O. (1997) "Market depth and order size", Lehrstuhl für Finanzierung Working Paper 97-05, Universit“at Mannheim.

[27] Kirman, A. (1983)

"Communication in markets: a suggested approach" Economics Letters, 12, 1-5.

[28] Kirman A., Oddou C. \& Weber S. (1986) "Stochastic communication and coalition formation" Econometrica, 54, 129-138.

[29] Kirman, A. (1996)"'Interaction and markets", GREQAM Working Pa- per.

[30] Lakonishok J., Shleifer A. \& Vishny R. (1992)"'Impact of institutional investors on stock prices", Journal of financial Economics, 32, 23-44. 


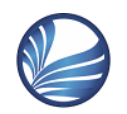

ELK

Asia Pacific Journals

www.elkjournals.com

[31] Levy, M. \& Solomon, S. (1997)'New Evidence for the Power Law Dis- tribution of Wealth" Physica A, 242, 90-94.

[32] R. Mantegna \& H. E. Stanley Nature, 376, 46-49 (1995).

[33] Orl'ean, A. (1995)'Bayesian interactions and collective dynamics of opinion" Journal of Economic Behavior and Organisation, 28, 257-74.

[34] Pagan, A. (1996)"The econometrics of financial markets" Journal of Empirical Finance,3, 15-102.

[35] Scharfstein D.S. \& Stein J.C. (1990)"Herd behavior and investment"American Economic Review, 80, 465-479.

[36] Sethna J.P., Dahmen K., Kartha S., Krumhans J.A., Roberts B.W. \& Shore J. (1993) "Hysteresis and hierarchies: dynamics of disorderdriven first-order phase transformations" Phys. Rev. Lett., $70,3347$.

[37] Sethna J.P., Perkovic O. \& Dahmen, K. (1997)'Hysteresis, avalanches and Barkhausen noise" to appear in Scale invariance and beyond, Proceedings of the CNRS Workshop on Scale Invariance (Les Houches, France), March 1997.

[38] Shiller R. (1989) Market volatility Cambridge, MA: MIT Press.

[39] Topol, R. (1991)"Bubbles and volatility of stock prices: effect of mimetic contagion" Economic Journal, 101, 786-800.

[40] Trueman, B. (1994)"Analysts forecasts and herding behavior" Review of financial studies, 7 (1), 97-124. 